\title{
Review of: "An account of Colletotrichum species associated with anthracnose of Atractylodes ovata in South Korea based on morphology and molecular data"
}

\section{GIL SANTOS}

Potential competing interests: The author(s) declared that no potential competing interests exist.

The article "An account of Colletotrichum species associated with anthracnose of Atractylodes ovata in South Korea based on morphology and molecular data " provides important information regarding the description of Colletotrichum species associated with the $A$. ovata plant in Korea. Although the methodology used in the morphological and molecular characterization are correct, I believe that the collection of a total of 30 leaves in five farms is not enough for a good sampling, since a great diversity of Colletotrichum species associated with this plant was found. In research that assesses the prevalence of pathogens and the identification of dominance of species, it is important to have a good sampling in the regions where the host plant grows. Another observation I can make about this study is about the method of inoculation that was done on detached leaves. Although it's not completely wrong and it can also be used, I prefer to inoculate the conidia suspension directly on the leaves of plants grown in pots. It allows us to have a best answer and view of the natural relationship between the pathogen and the host and a real response to the activation of the plant's resistance mechanisms. This procedure certainly interferes with the size of the lesions. Figure 19 should also include the results of lesions formed without wounds, since some species caused disease in leaves even without wounds. In the discussion, according to the results obtained, the authors should suggest, in a preliminary way, some strategies to control the disease according to the management of the culture adopted by the producers. 